$68^{\text {th }}$ International Astronautical Congress (IAC), Adelaide, Australia, 25-29 September 2017.

Copyright 2017 by German Aerospace Center (DLR). Published by the IAF, with permission and released to the IAF to publish in all forms.

IAC-17,A6,IP,5,x37746

\title{
STAR-C: Towards a transportable Laser Ranging Station
}

\author{
T. Hasenohr ${ }^{\mathrm{a} *}$, L. Humbert ${ }^{\mathrm{b}}$, D. Hampf ${ }^{\mathrm{c}}$, W. Riede ${ }^{\mathrm{d}}$, \\ a, b, c, d German Aerospace Center (DLR), Institute of Technical Physics, Pfaffenwaldring 38-40, 70569 Stuttgart, \\ Germany, \\ a thomas.hasenohr@dlr.de, ${ }^{\mathrm{b}}$ leif.humbert@dlr.de, ${ }^{\mathrm{c}}$ daniel.hampf@dlr.de, ${ }^{\mathrm{d}}$ wolfgang.riede@dlr.de, \\ * Corresponding Author
}

\begin{abstract}
For surveillance of the Low Earth Orbit (LEO) population the orbits of resident space objects, such as satellites but also space debris, have to be known very accurately. Precise measurements of the slant range to these targets can improve the orbit determination. These measurements can be performed by ground based satellite- and space debrislaser ranging.

At the Institute of Technical Physics at the German Aerospace Center new optical technologies are developed and investigated for the detection, determination and ranging of space debris. In this context, a transportable laser ranging station STAR-C (Surveillance, Tracking and Ranging-Container) for laser ranging of space debris in LEO will be set up. A standard $20 \mathrm{ft}$ ISO container, which can be operated autonomously with a $14 \mathrm{kVA}$ generator, contains all the scientific equipment and work places. The implemented laser ranging system raises above the roof of the container within less than 1 min by a lifting platform. A transmitter- and a 17 " receiver telescope are mounted on an agile altazimuth mount with an encoder resolution of 26 bit which gives an angular resolution of 1/50 arcseconds. In order to use the needed pulse energies for a ranging to space debris the STAR-C system will guide laser pulses through a Coudé train from the laser to the transmitter telescope. A Nd:YAG system at a wavelength of $1064 \mathrm{~nm}$ will be used as a laser pulse source. At repetition rates of about $500 \mathrm{~Hz}$ the pulse energy shall be higher than $50 \mathrm{~mJ}$ for pulse durations (FWHM) smaller than $5 \mathrm{~ns}$. The transportability of this system allows measurements from all over the world or filling gaps in a global network of space surveillance systems.

This paper presents the transportable laser ranging station STAR-C and reports on the progress in setting up this system. In addition, the difficulties of pointing due to the tilt of the lifting platform will be discussed.
\end{abstract}

\section{Keywords: Satellite Laser Ranging, Space Debris, SSA, Low Earth Orbit, Ground Station}

\section{Nomenclature}

$\varphi_{\mathrm{x}} \quad$ Platform-inclination along the long side of the container

$\varphi_{\mathrm{y}} \quad$ Platform-inclination along the short side of the container

\section{Acronyms/Abbreviations}

Automatic Dependent Surveillance - Broadcast (ADS-B)

Coordinated Universal Time (UTC)

Field of View (FOV)

Global Positioning System (GPS)

Long Term Evolution (LTE)

Low Earth Orbit (LEO)

Orbital Objects Observation Software (OOOS)

Resident Space Objects (RSO)

Surveillance, Tracking and Ranging-Container (STAR-C)

Satellite Laser Ranging (SLR)

Two Line Element (TLE)

\section{Introduction}

The increasing number of active satellites (e.g. mega constellations) leads to an expected growth of the space debris population. Even today space debris is one of the major threats for active satellites and manned space missions. A collision in space does not only affect the operational work of a satellite. It can also cause a sudden expansion of the debris population, which increases the threat for spacecraft even more.

In 2007 the Chinese anti-satellite test and the accidental collision of the active Iridium 33 and the dysfunctional Cosmos 2251 in 2009 increased the number of debris in LEO by about $60 \%$ [1]. These two events are responsible for four of six close approaches of debris to the International Space Station in just 12 months (April 2011 to April 2012) where four collision avoidance maneuvers were necessary [2].

Therefore, the Low Earth Orbit (LEO) population has to be monitored closely in order to avoid collisions and to enhance the predictions of approaches of space debris to active spacecraft. Precise spatial measurements of the trajectory of a target and a subsequent orbit determination can be performed by laser ranging 
stations. These systems can improve the orbits contained in catalogs of resident space objects (RSO) like the one provided by the U.S. Space Surveillance Network (SSN). This catalog contains over 40.000 RSOs of which more than 17.200 are still in orbit [3]. The included LEO-data are mainly based on Radar data, which is most common for LEO surveillance i.a. due to the independence of weather conditions and the possibility to detect many targets at once. However, the precise measurements of the slant range performed by satellite- and space debris- laser ranging can make a significant contribution to the accuracy of such a catalog.

In the following, this paper presents the transportable Laser Ranging Station "Surveillance, Tracking and Ranging-Container" (STAR-C) and the hardware used. The issues of a pointing model of a telescope mounted on a lifting platform are discussed based on measurements in the second section. Finally, an outlook is given what the next steps in setting up this system will be and what the objectives are.

\section{Surveillance, Tracking and Ranging-Container}

This section describes in detail the specifications, the current set-up and the main features of the STAR-C with the goal to give an overview of the system but also to show its potential.

\subsection{General Information}

In 2016 the DLR in Stuttgart instructed the company WTW Anlagenbau GmbH with developing and settingup an alt-azimuth telescope mount for a space debris ranging station. During the drafting process the idea was born to integrate this mount onto a lifting platform in a container for transportation to arbitrary sites.

The whole system weighs about $10 \mathrm{t}$ and is contained in a $20 \mathrm{ft}$ ISO container, shown in figure 1. Due to this, it is easy to transport the station for every shipping company by truck, train, ship or even by airplane. STAR-C's interior is separated into two rooms. First, the "operator room", where there is room for up to three operators. An air conditioning unit, an electric cabinet and a further cabinet for equipment like IT-Hardware and supply modules for the laser system are located here as well. The second room is the "telescope room". A structure, which can raise a platform including the telescope mount above the roof, is located here. Due to its importance this room is described in subsection 2.2 in more detail. Both rooms are fully separated which has the advantage that the operator is not affected by the ambient temperatures or the laser light.

An external power point can be used to power the system, but to ensure an independent operation the STAR-C can also be powered by a $14 \mathrm{kVA}$ generator.
Furthermore, the current laser system as well as the planned one will not need an external water supply. The internet connection is provided by a Long Term Evolution (LTE) connection for downloading current TLE data and in future for data exchange with other satellite laser ranging (SLR) stations.

A self-developed software, called Orbital Objects Observation Software (OOOS) [4], will control the mount and all equipment for satellite and space debris ranging tasks in future.

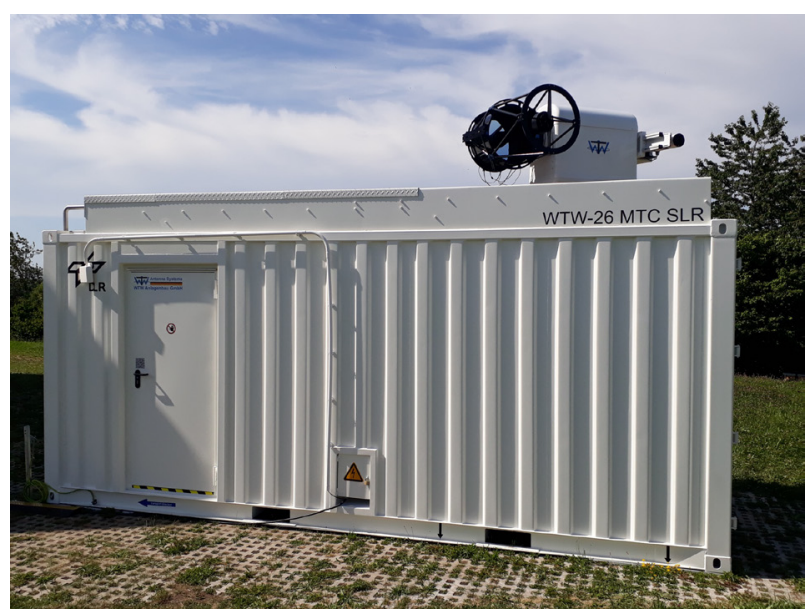

Fig. 1: Surveillance, Tracking and Ranging-Container (STAR-C) with the raised telescope system.

\subsection{Telescope room}

The "telescope room" contains a $4.0 \mathrm{t}$ metal frame, which supports a platform carrying the telescope mount and a housing for instrumentation. It is also used as vibration mitigation in case the telescope is affected by wind. In order to ensure no transfer of vibrations from the inside of the container (e.g. caused by the operator) to the telescope mount the frame is mechanically decoupled from the container itself. This is done by four legs which are reaching through the floor of the container to the ground and are lifting the whole frame.

Figure 2 shows on the left hand side the offline state where the lifting platform is retracted and the telescope mount with its equipment is sheltered by the container. In order to start a measurement, this platform can be raised and guided by two mandrels after opening a retractable roof. The maximum payload including the telescope mount can be up to $1500 \mathrm{~kg}$. This makes it possible to carry the laser and the other optical and timing equipment on this platform in addition to the mount. 

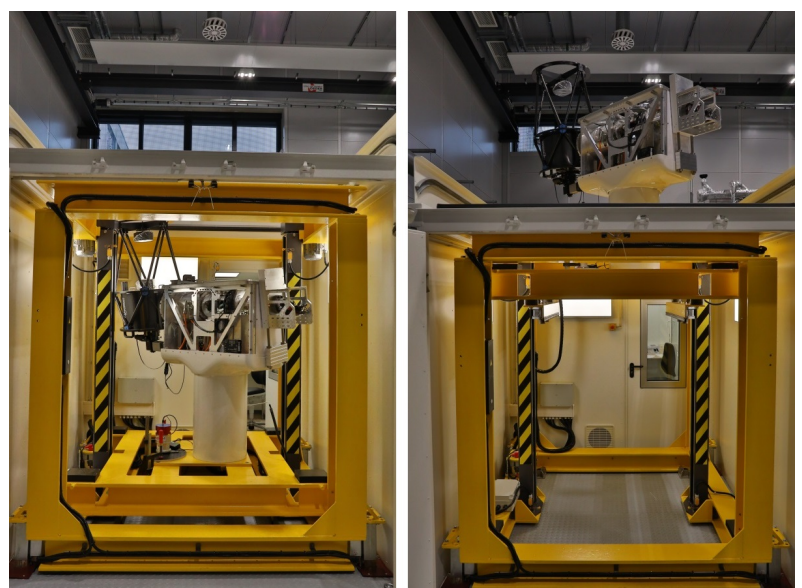

Fig. 2: Telescope room containing the lifting platform of the STAR-C. Left: Telescope-Mount is offline and sheltered by the closed sliding roof. Right: TelescopeMount is raised above the sliding roof and is in online state.

The two mandrels are powered by electric drives controlled by a frequency converter to ensure a smooth motion during raising and lowering. Figure 2 shows on the right hand side the top position. Here, the lifting platform is pressed onto the frame, which serves as absorber, to transfer the vibrations affecting the telescope.

\subsection{Telescope mount}

An altazimuth mount designed by WTW Anlagenbau $\mathrm{GmbH}$ is equipped with a transmitter- and a receiver-telescope and is shown in figure 3 . Its fast rotation velocities up to $15 \%$, allows choosing quickly between targets. Due to this it will also be possible to measure the slant range of multiple passing targets while switching back and forth between them. Slip rings at the azimuth axis obviate a "side flip" while the elevation axis is equipped with a slip rings as well in order to avoid torsion of cables. Therefore, the rotation range is $0^{\circ}$ to $>360^{\circ}$ for the azimuth axis while the elevation axis is limited from $0^{\circ}$ to $85^{\circ}$ at the current version of the mount. This is due to an interconnection of the transmitter telescope to the elevation-axis-drive where the receiving telescope is mounted directly.

Measurements of the rotation positions are done by absolute encoders with a resolution of 26 bit $(0.1 \mu \mathrm{rad})$, which makes a reference run at the beginning of a satellite laser ranging measurement unnecessary. Inside the axes a Coudé train allows guiding high energy laser pulses from the laser source mounted on the lifting platform to the transmitter telescope. Beam stabilization and beam control will be set up directly inside the mount and will be adjusted directly during laser ranging [5].

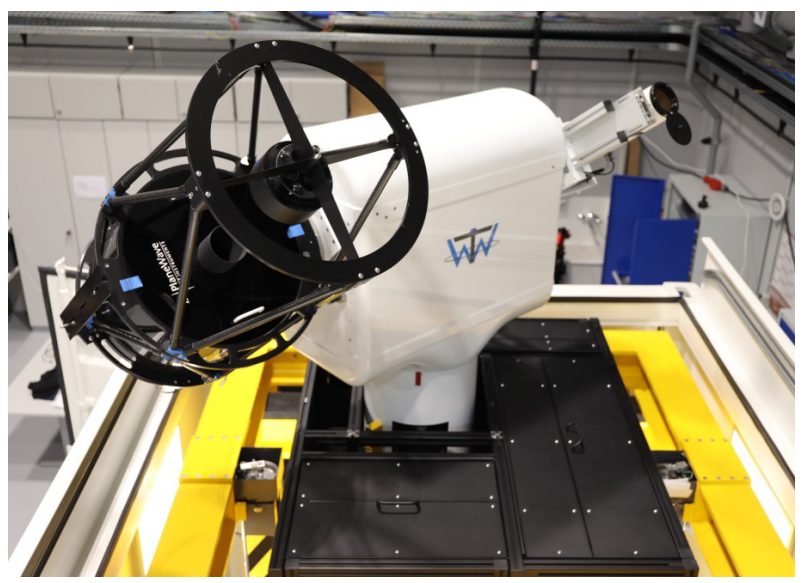

Fig. 3: Altazimuth mount of the STAR-C system equipped with a 17 "-telescope for receiving the laser pulses and a 4 "-Galilei-telescope as laser transmitter.

\subsection{Measuring equipment}

For laser ranging to satellites or space debris reliable and simultaneous operation of many instruments is necessary.

An overview of the current set-up for initial passive optical tests and to achieve laser ranging to our calibration site and to cooperative orbiting targets is given in the following. Except the transmitter telescope, all devices are commercial off the shelf components and are available from various suppliers. However, not all of them are already implemented in the current set-up and in use for testing. A summary of the components with full names and further information is given in table 1.

For passive optical tracking of a TLE-object, a 17 " receiver-telescope with a focal length of $2900 \mathrm{~mm}$ is used. A field of view (FOV) of $0.32^{\circ} \times 0.27^{\circ}$ is given due to the large image sensor of an sCMOS camera, while the resolution is about 0.5 arcseconds per pixel. The control software [4] will fix the object at a certain position in the FOV, which occurs as dot during the track at the image center. This setup is identical to the one used at the research observatory at the Uhlandshöhe, Stuttgart developed by the DLR [6], which is able to track CubeSats in LEO during good conditions. Therefore the same results are expected at the STAR-C. However, it is currently limited for tracking visible objects only during dusk or dawn.

While tracking, laser pulses generated by a Nd:YAG laser source at a wavelength of $1064 \mathrm{~nm}$ are guided along the Coudé train to the 4" transmitter telescope. This emits the light to the target. Each laser pulse which is guided along the Coudé train is time tagged by a high precision event timer triggered by a photo diode. In order to manage high laser pulses the Coudé train has a free aperture of $36 \mathrm{~mm}$, while the transmitter telescope is a Galilei design with lenses coated for the fundamental wavelength of a Nd:YAG laser. 
$68^{\text {th }}$ International Astronautical Congress (IAC), Adelaide, Australia, 25-29 September 2017.

Copyright 2017 by German Aerospace Center (DLR). Published by the IAF, with permission and released to the IAF to publish in all forms.

Table 1: Equipment used for Laser Ranging and as environment sensors in the STAR-C

\begin{tabular}{llll}
\hline Instrument & Manufacturer & Device & Information \\
\hline Telescope & Planewave & CDK17 & 17” aperture \\
Camera & Andor & Zyla & sCMOS \\
Detector & Aurea Technologies & SPD_OEM_NIR & InGaAs- SPAD \\
Transmitter & Self-made & - & 4"Galilei- Design \\
Laser & CryLas & DSS 1064-3000 & 532/1064nm; 1000Hz; $\sim 60 \mu \mathrm{J}$ \\
Beam Control & Newport & Guidestar II & - \\
Event timer & Eventtech & A033-ET & Resolution $=5 \mathrm{ps}$ \\
GPS-sync & Meinberg & GPS180PEX-HQ & - \\
ADS-B & Modesbeast & Radarcape & $1090 \mathrm{MHz}$ \\
$\begin{array}{l}\text { Weather sensor } \\
\text { Inclination }\end{array}$ & Diffraction Ltd. & Boltwood Cloud Sensor II & Sky conditions, temperature, wind, humidity \\
sensor & Wyler AG & Zeromatic 2/2 & Precision \pm 1 arcseconds; $2 \mathrm{~Hz}$ \\
\hline
\end{tabular}

The returning laser photons are collected by the receiver-telescope and detected by an InGaAs single photon detector, while the remaining light will be reflected to the tracking camera by a dichroic beam splitter. This detector triggers again the event timer, which now has the time of flight with a resolution of $5 \mathrm{ps}$. The relative time of flights are synchronized to the Coordinated Universal Time (UTC) by a Global Positioning System (GPS) receiver to get an absolute correlation to the target.

A cloud sensor for taking weather data and an Automatic Dependent Surveillance - Broadcast (ADS-B) antenna will monitor the environment during operation. This antenna receives the transponder signals of the aircrafts at the surrounding airspace and can be used for closing a shutter to prevent emitting laser light in the direction of an aircraft. More sensors for airspace surveillance will be implemented in future before using a space debris laser at full power.

\section{Tilt of the lifting platform}

The metal frame which carries the lifting platform is supported by four feet and has a further task besides the decoupling of the telescopes from the container. It is possible to make an initial adjustment of the inclination by those feet. Each of them can be extended separately. This guarantees a horizontal alignment better than $0.3^{\circ}$ in accuracy. Thereby, minor inclinations of an arbitrary site can be balanced.

However, the disadvantage of a movable platform where the telescope mount is carried is its tilt during operation, which has to be adjusted for a successful measurement. Two reasons are causing this tilt in the current set-up: First, the telescope mount is not centered at the lifting platform due to the large receiving telescope and the smaller transmitter telescope. Second, the platform is pressed to the frame and is not bolted or fixed otherwise in the raised state. There are four attachment points between the platform and the frame where currently buffers out of rubber are attached. The beforehand mentioned inclination changes every time when the platform reaches its upper position due to a different contact pressure and whenever the telescope is moving because of the changing load distribution on the platform.

The following subchapters show measurements of the inclination of the platform and discuss the results. Upgrade-possibilities for bypassing these problems are given in addition.

\subsection{Measurements and Results}

A high precision inclination sensor with a resolution of \pm 1 arcseconds is mounted on the lifting platform to measure the inclination of it at its upper level. This sensor will be used to adjust the specific telescope pointing model. Two aspects are examined in the following: First the tilt of the platform by rotating the azimuth axis during various elevations and second the initial tilt of the platform after lowering and raising it at different days. For both measurements the inclination sensor gives the inclination along the long side of the container $\varphi_{\mathrm{x}}$ and across the short side of the container $\varphi_{\mathrm{y}}$.

Figure 4 shows the inclinations in arcseconds along the container (top) and across (bottom) of different relative azimuth angles in degree. For this dynamical measurement the telescope was rotating three times in azimuthal direction at a speed of $1 \%$ with a fixed elevation. Measurements were taken with a rate of $2 \mathrm{~Hz}$ at elevations of $10^{\circ}$ (blue $\diamond$ ), $30^{\circ}$ (yellow $\circ$ ), $50^{\circ}$ (green $\Delta$ ) and $70^{\circ}($ red +$)$. The peak to peak amplitudes are about 42 "for $\varphi_{\mathrm{x}}$ while the amplitudes in $\varphi_{\mathrm{y}}$ are about 90 ". During various elevations the amplitudes of the inclination are not changing which shows that the elevation itself does not have an influence to the tilt of the platform. A shift could only occur if the telescope itself would not be balanced on the mount. The shifting inclinations so far can be compensated by a standard pointing model. 

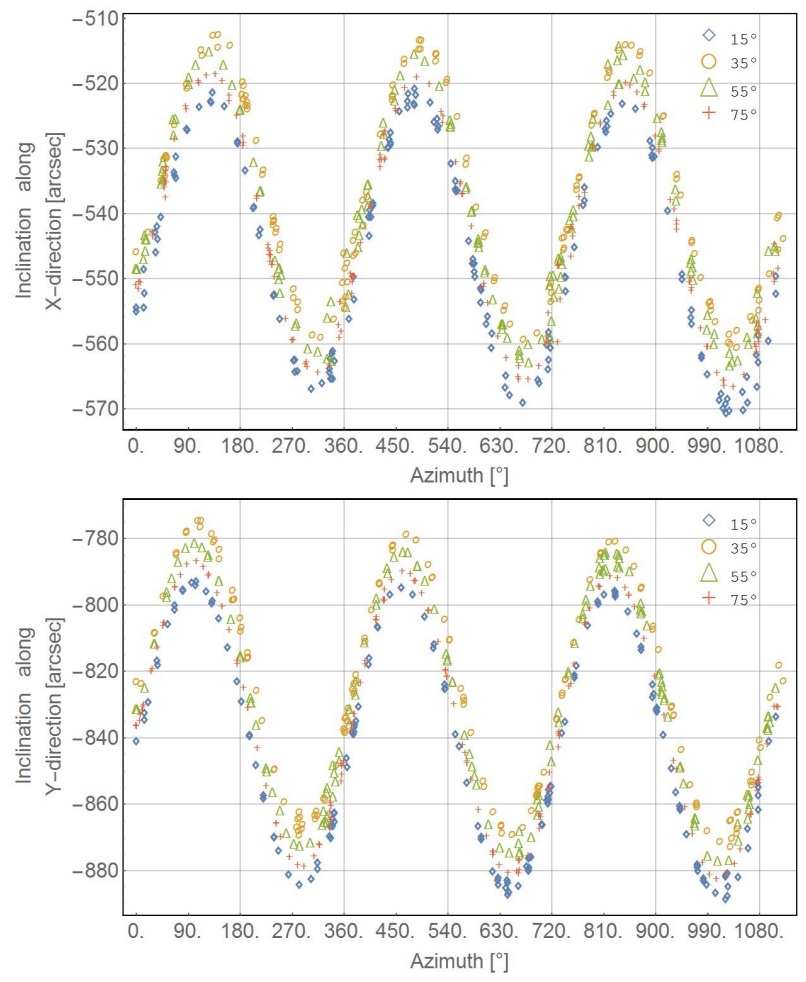

Fig. 4: Measurements of the inclination (top along, bottom across the container) of the STAR-C lifting platform during rotation in azimuth of the telescope. The rotational speed is $1 \%$ s at different elevations of the telescope.

However, all measurements show a similar trend in the shift of the inclination for various elevations $\left(15^{\circ}\right.$, $35^{\circ}, 55^{\circ}$ and $75^{\circ}$ ). An explanation is provided by figure 5. During the measurements at sunshine the whole system was heating up. The increasing temperature affects the buffers out of rubber, which are getting softer and can be more compressed. This drifting effect is not predictable and requires a continuous surveillance of the state of the lifting platform.

Whenever the system goes online the telescope will be raised and the lifting platform presses against the frame. It stops as soon as a limit switch signals the raised state. This measurement is not accurate which leads to different tilts every time. Figure 6 shows these different inclinations in $\varphi_{\mathrm{x}}$ (top) and $\varphi_{\mathrm{y}}$ (bottom) on three different days at elevations of $15^{\circ}$. The measurement shows the different initial inclinations of each measurement in both directions $\left(\varphi_{\mathrm{x}}, \varphi_{\mathrm{y}}\right)$. However, a correlation of the deviation of the tilts in $\varphi_{\mathrm{x}}$ and $\varphi_{\mathrm{y}}$ is not noticeable. In $\varphi_{x}$ the outlier was at the second measurement on August $15^{\text {th }}$ while in $\varphi_{\mathrm{y}}$ it goes with the measurement on August $14^{\text {th }}$. According to the measurements deviations larger 20 " could occur as initial inclination shift during a restart of the system and has to be compensated in advance to laser ranging.
However, the subsequent rotation measurements show that the initial inclination does not change during a movement of the telescope.

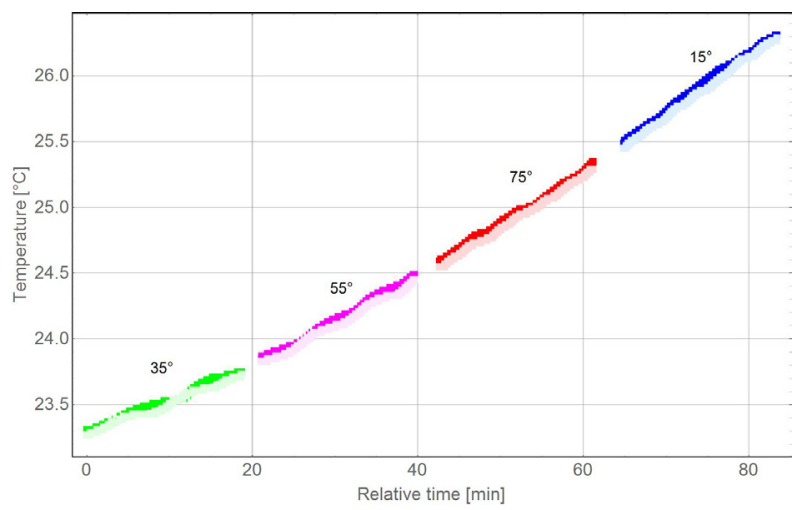

Fig. 5: Temperature of the lifting platform during the inclination measurements while rotating the azimuth axis at different elevations. The temperatures of the inclination sensor along the container are in darker and for the sensor across the container are in lighter colors.
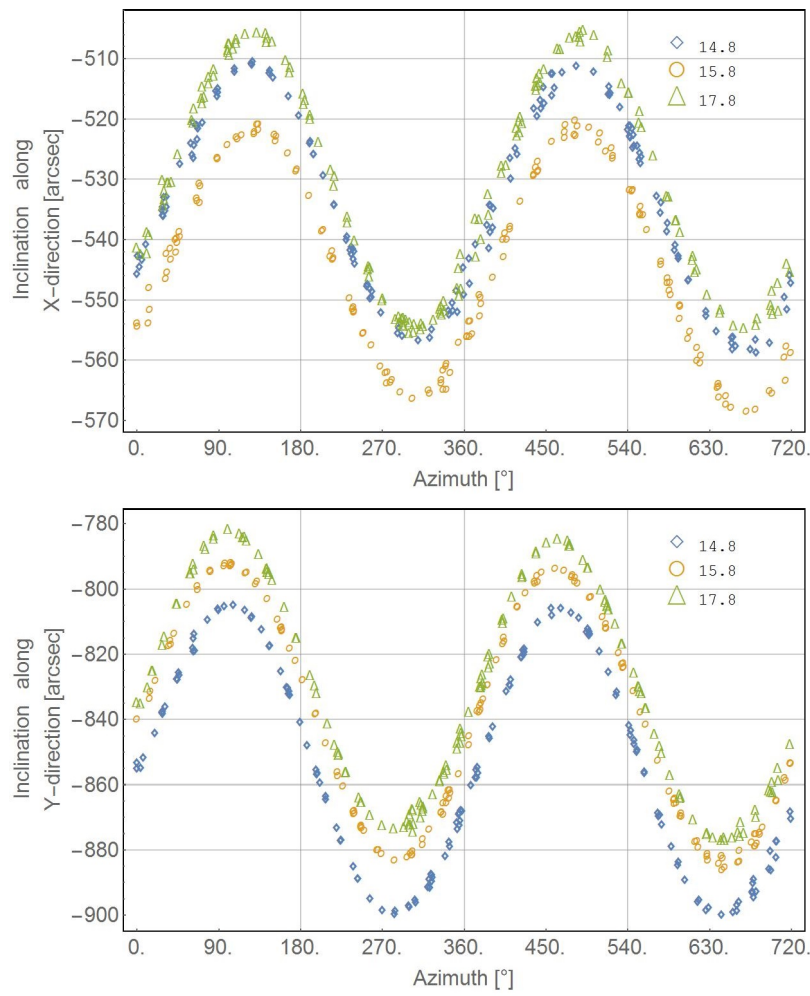

Fig. 6: Measurements of the inclination (top along the long side, bottom across the short side of the container) of the STAR-C lifting platform during rotation in azimuth of the telescope on three different days of measurement. Between the measurements the lifting platform was lowered. 


\subsection{Discussion}

The measurements in subsection 3.1 show the inclinations of the lifting platform which have a correlation to the tilt of the telescope mount itself. In order to avoid an influence of them to the measurements they have to be take into account by the pointing model. Therefore a continuous measurement of the inclinations can be used as offsets by the pointing model during the observation.

In order to bypass the problems of the tilting platform various methods are possible: The first possibility would be to replace the buffer made of rubber by metal plates. Another advantage in doing that is the improved transfer of the vibrations from the platform to the frame. This upgrade is planned at the STAR-C as soon as further upgrades to enable daylight tracking will be realized. Another method would be bolting the platform at the upper position. However, it has the disadvantage that this has to be done every time again when the system goes online. Between the measurements the telescope is retracted where it is protected against environmental influences like rain. A third, but most expensive, possibility would be upgrading the whole lifting structure. Currently, just two mandrels are supporting the platform. This could be extended by another two. With four mandrels (one at each corner) and an inclination sensor a control system could adjust misalignments online.

However, in the current state the large FOV of the tracking telescope in combination with the closed loop for chasing visible target makes an upgrade not necessary. When the STAR-C shall be able for daylight tracking in future, the lifting structure has to be upgraded and an advanced pointing model has to be used.

\section{Outlook}

The STAR-C is in an early stage of construction and many systems are not implemented or tested, yet. The next steps towards space debris laser ranging are to generate an adaptable pointing model and to test the accuracy of the passive optical tracking of known satellites. Afterwards, the set-up of the Coudé train with its beam stabilization and the timing equipment will be implemented. As soon as all systems are tested a first laser ranging to a terrestrial calibration site will be accomplished. The current laser allows satellite laser ranging to cooperative targets after the calibration, as well. In order to ensure the ability of space debris laser ranging the laser system will be upgraded in spring 2018. A new $1064 \mathrm{~nm}-$ laser is under development from neoLASE GmbH which will have a higher peak power while having short pulse durations. Hence, the STAR-C will still be independent as the new laser system does not need fresh water for cooling. For safety reasons further equipment for scanning of the environment will be implemented as well. A long term goal will also be the upgrade of the system to enable remote control.

\section{Conclusions}

First tests of the STAR-C look promising. Measurements of the tilt of the lifting platform show that the resulting inclinations have no critical influence for laser ranging to visible targets. For daylight tracking tests with an advanced pointing model have to be accomplished. However, this is not a requirement at this state.

The unique feature of transportability has not only the advantage that such a system can be moved wherever it is currently needed. It would also be possible to set it up and test it in a known environment and taken afterwards to its final location. This could decrease the costs of building up a global network.

In conclusion, the STAR-C system and maybe multiple duplications could contribute to the installation of a global network for space surveillance or geodetic purposes.

\section{Acknowledgements}

The authors would like to thank WTW Anlagenbau $\mathrm{GmbH}$ for their technical support and cooperation during the time of development. In addition we want to thank the German Meterological Office (Deutscher Wetterdienst) in Stuttgart for their hospitality during the first tests.

\section{References}

[1] J.-C. Liou, editor, "Update on Three Major Debris Clouds", Orbital Debris Quarterly News, 14(2), April 2010, www.orbitaldebris.jsc.nasa.gov.

[2] J.-C. Liou, editor, "Increase in ISS Debris Avoidance Maneuvers", Orbital Debris Quarterly News, 16(2), April 2012, www.orbitaldebris.jsc.nasa.gov.

[3] P. Anz-Meador, editor, "Top Ten Satellite Breakups Reevaluated", Orbital Debris Quarterly News, 20(1\&2), January/April 2016, www.orbitaldebris.jsc.nasa.gov.

[4] D. Hampf, et al., "OOOS: A hardware-independent SLR control system", ILRS Technical Workshop 2017, in preparation 2017

[5] L. Humbert, et al., "Design and commissioning of a transportable laser ranging station STAR-C", in 2017 AMOS Conference, in preparation 2017

[6] D. Hampf, et al., „First successful satellite laser ranging with a fibre-based transmitter", Advances in Space Research, Volume 58, 2016 